\title{
QED Corrections to the Electromagnetic Abraham Force
}

\section{Casimir Momentum of the Hydrogen atom?}

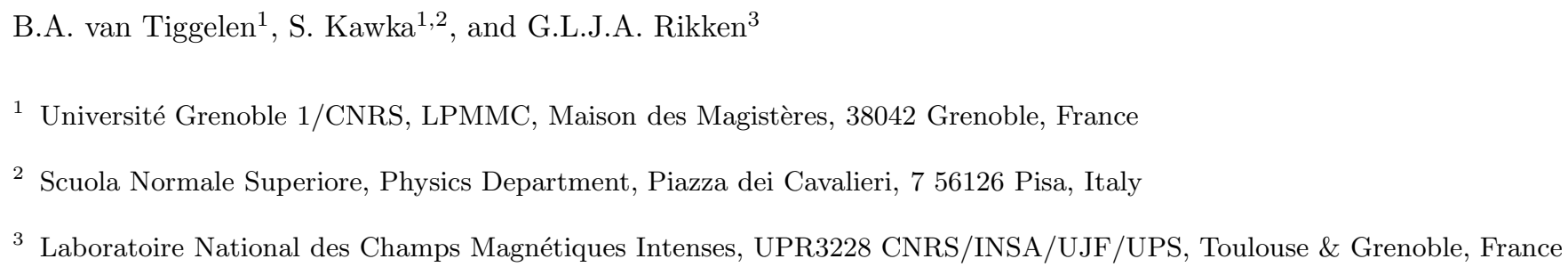

Abstract. We develop a QED approach to find the contribution of the quantum vacuum to the electromagnetic Abraham force. Semi-classical theories predict diverging contributions from the quantum vacuum. We show that the divergencies disappear by Kramers-Bethe mass-renormalization. The finite remainder is compared to the relativistic corrections to the Abraham force. This work generalizes an earlier paper [1], dedicated to the harmonic oscillator, to the hydrogen atom and corrects two subtle errors.

\section{Introduction}

The Abraham force refers to the small force exerted by time-dependent electromagnetic fields on neutral matter. Despite our complete knowledge on classical electromagnetism, a longstanding controversy exists about the precise expression of this force, given macroscopic, phenomenological constants such as dielectric constant and magnetic permeability. This so-called "Abraham-Minkowski" controversy stems mainly from the fact that the macroscopic Maxwell's equations seem to favor a radiative momentum density $\mathbf{D} \times \mathbf{B}[2]$ (the Minkowski version), whereas the Send offprint requests to: microscopic version leads to $\varepsilon_{0} \mathbf{E} \times \mathbf{B}$ (the Nelson version [3]). In addition, neither one of them is in general equal to $\varepsilon_{0} \mu_{0} \mathbf{E} \times \mathbf{H}$, that is radiative momentum does not always seem to be just equal to energy flow $\mathbf{S}=c_{0}^{-1} \mathbf{E} \times \mathbf{H}$, divided by $c_{0}$ (the Abraham version, also advocated by Jackson [2]). The different versions lead to different expressions for the Abraham force [4].

Following Nelson [3, it is instructive to calculate directly the force density $\mathbf{f}=\partial_{t}\left(\rho_{m} \mathbf{v}\right)$ exerted on the matter, given by the Lorentz force $\mathbf{f}=\rho_{q} \mathbf{E}+\mathbf{J}_{q} \times \mathbf{B}$ with $\rho_{q}=\nabla \cdot \mathbf{P}_{q}$ and $\mathbf{J}_{q}=\nabla \times \mathbf{M}_{q}-\partial_{t} \mathbf{P}_{q}$ the bound charge 
density and bound charge current density. For nonmagnetic materials without free charges this leads to

$$
\partial_{t}\left(\rho_{m} \mathbf{v}-\mathbf{P}_{q} \times \mathbf{B}\right)=\nabla \cdot \mathbf{U}-\frac{1}{2}\left(\nabla \varepsilon_{r}\right) \varepsilon_{0} \mathbf{E}^{2}
$$

with $\mathbf{U}$ a second rank stress tensor specified elsewhere $[5]$. Since the space integral of $\nabla \cdot \mathbf{U}$ vanishes, and so does the last term in a sufficiently homogeneous field, the force on the atom is equal to the classical "Abraham force",

$$
\mathbf{F}_{A}=\partial_{t} \int d \mathbf{r}\left(\mathbf{P}_{q} \times \mathbf{B}\right)=\varepsilon_{0}\left(\varepsilon_{r}-1\right) V \partial_{t}(\mathbf{E} \times \mathbf{B})
$$

Only a few observational claims exist, such as the work by Walker [6], and our recent work on gases [7].

Since a few years several authors have discussed the possibility of a contribution to the Abraham force stemming from the electromagnetic quantum vacuum. In the remainder we will refer to the momentum associated with this QED contribution to the Abraham force as "Casimir momentum". Its existence seems even more controversial. The first approaches are based on a semi-classical analysis, i.e. classical for the matter, and quantum for the radiation. Following the early work in Ref. [8], Refs. [12] and [10] demonstrate that Casimir momentum can exist in socalled bi-anisotropic media. These are media where electric fields induce magnetic moments, and by symmetry, magnetic fields induce electric moments. All media can be made bi-anisotropic, for instance by moving them or by exposing them to crossed electric and magnetic fields. However, two major problems show up for Casimir momentum in such media. First, the end result diverges significantly in the UV and needs to be regularized, and the question is whether the effect survives the regularization. Secondly, the question about Lorentz-invariance arises, and more generally, the need to include relativistic effects. In a recent Reply [5] a fully Lorentz-invariant model was discussed in which Casimir momentum exists, although still with a diverging value.

In Ref. [8] the divergent frequency integral was solved by a simple cut-off frequency in the X-ray regime, as had previously been proposed by Schwinger 9 to calculate the Casimir energy of an oscillating bubble. Dimensional regularization was proposed by Brevik etal [11. When applied to Casimir momentum [12, one finds values inversely proportional to the sample size but still resulting in extremely small values for micro-sized media. In a recent work [1] we considered the quantum-mechanical harmonic oscillator exposed to quasi-static fields as well as to the quantum vacuum. We showed that Kramer's mass renormalization [13,14] eliminates the divergencies. Unfortunately, this work suffers from two errors that will be repaired in this work. In addition we shall consider the hydrogen atom with Coulomb interaction. This will allow us to conclude that relativistic effects are (here) much smaller, typically by a factor $m_{e} / M$.

\section{UV catastrophe in bi-anisotropic media}

The problem that occurs in bianisotropic media is most easily illustrated by considering an infinite, dispersionless dielectric medium moving at speed $\mathbf{v}$ with respect to the observer. Such medium is subject to the Fizeau 
effect and has a bi-anisotropic coupling tensor equal to $g_{i j}=\varepsilon_{0}\left(1-\varepsilon_{r}\right) \epsilon_{i k j} v_{k}$, i.e the magnetic field induces a polarization density equal to $\varepsilon_{0}\left(1-\varepsilon_{r}\right) \mathbf{v} \times \mathbf{B}$. It is straightforward to see that the quantum expectation value of the Abraham momentum density $\mathbf{P}_{\mathrm{A}}=-\mathbf{P}_{q} \times \mathbf{B}$ is given by $\left(2 \mathbf{v} / 3 c_{0}^{2}\right)\left(\varepsilon_{r}-1\right)\left\langle\mathbf{B}^{2} / \mu_{0}\right\rangle$ and is directed along the direction v. Defining the "Casimir inertial mass density" $\rho_{C}$ as $\mathbf{P}_{A}=\rho_{C} \mathbf{v}$ that in Eq. (11) adds up to the momentum density $\rho_{m} \mathbf{v}$ of "matter", gives

$$
\rho_{C}=\frac{2}{3} \frac{\hbar}{\pi^{3} c_{0}^{5}} \int_{0}^{\infty} d \omega\left(\varepsilon_{r}-1\right) \omega^{3}
$$

This integral diverges. Usually the divergence of Casimir energy poses no problem as long since the observable forces are finite. The Casimir energy between two ideal mirrors is known to have a finite inertial mass [15. Being an observable quantity itself, the diverging inertial mass density $\rho_{C}$ does pose a problem. We can identify $\left\langle\mathbf{E} \cdot \mathbf{P}_{q}\right\rangle=$ $\int d \omega\left(\varepsilon_{r}-1\right) \hbar \omega^{3} / \pi^{3} c_{0}^{3}$ as the potential energy density of the quantum vacuum. Indeed, what we might expect to find for $\rho_{C}$ is (the inertial mass due to) the binding energy associated with Coulomb, Van-der-Waals and Casimir Polder forces [16] that can be viewed as longitudinal and transverse degrees of freedom of the vacuum electromagnetic field, and which are also known to find their way to the dielectric constant [20]. Apart from the divergence, the front factor $2 / 3$ in Eq. (3) is also strange, and reminiscent of the problem of electromagnetic self-mass of the electron [2].

If we assume free electron optical dispersion $\left(\varepsilon_{r}=\right.$ $\left.1-n_{e} e^{2} / \varepsilon_{0} m_{e} \omega^{2}\right)$ at high frequencies [2], the divergence of Casimir momentum will still persist as $\int d \omega \omega$. If we as- sume that frequencies larger than $\pi / r_{e}$ do not contribute by some unknown physical principle (valid at length scales smaller than the free electron radius) the mass density (3) would typically be equal to $n_{e} m_{e} / \alpha$, i.e. a factor $1 / \alpha$ larger than the actual mass density of the electrons. It seems likely that this mass is already counted in the physical values attributed to the masses of particles, as was already suggested in literature [14]. In this paper we shall validate this argument explicitly for the hydrogen atom, both for the Fizeau effect and for the magneto-electric effect. For the first this is quite naturally suggested by Eq. (3). For magneto-electric materials the quantum vacuum gives a similar UV divergence [8] for which mass renormalization is much less obvious.

In the following we consider the quantum mechanics of a hydrogen atom exposed to crossed, quasi-static electromagnetic fields. We calculate the total (pseudo-) momentum of the atoms and identify diverging terms when coupled to the quantum vacuum. We regularize the infinities, and finally find the finite contribution of the quantum vacuum to the momentum, into lowest order of the fine structure constant $\alpha$.

\section{A moving hydrogen atom coupled to EM quantum vacuum and external fields}

We consider a hydrogen atom moving at non-relativistic speed, in crossed electric and magnetic fields $\mathbf{E}_{0}$ and $\mathbf{B}_{0}$ respectively. Particle 1 is the proton, particle 2 the electron. We shall use the reduced coordinates $\mathbf{R}=\left(m_{1} \mathbf{r}_{1}+\right.$ 
$\left.m_{2} \mathbf{r}_{2}\right) / M$ and $\mathbf{r}=\mathbf{r}_{1}-\mathbf{r}_{2}$ for the center of mass position and the interparticle distance, with conjugate momenta $\mathbf{P}=\mathbf{p}_{1}+\mathbf{p}_{2}$ and $\mathbf{p}=\mu\left(\mathbf{p}_{1} / m_{1}-\mathbf{p}_{2} / m_{2}\right)$. The presence of an external stationary magnetic field $\mathbf{B}_{0}$ will be treated in the Coulomb gauge and it is instructive to make the unitary transformation $U=\exp \left[i e\left(\mathbf{B}_{0} \times \mathbf{R}\right) \cdot \mathbf{r} / \hbar\right]$ that conveniently removes $\mathbf{R}$ from the Hamiltonian [17. We shall write $H=H_{0}+H_{\text {rel }}+H_{F}+W$ and denote with $\tilde{H}=U^{*} H U$ the transformed Hamiltonian. The nonrelativistic, transformed Hamiltonian of the atom is given by

$$
\begin{aligned}
\widetilde{H}_{0}= & \frac{1}{2 \mu}\left(\mathbf{p}+\frac{\Delta m}{M} \frac{e}{2} \mathbf{B}_{0} \times \mathbf{r}\right)^{2}+\frac{1}{2 M}\left(\mathbf{P}-e \mathbf{B}_{0} \times \mathbf{r}\right)^{2} \\
& -\frac{e^{2}}{4 \pi \varepsilon_{0} r}-e \mathbf{E}_{0} \cdot \mathbf{r}
\end{aligned}
$$

with $\Delta m=m_{1}-m_{2}>0$ [1]. This transformed Hamiltonian commutes clearly and conveniently with the total conjugated momentum $\mathbf{P}$, even when $\mathbf{E}_{0}$ depends on time, meaning that (when transformed back) the pseudo momentum $\mathbf{Q}=U^{*} \mathbf{P} U=\mathbf{P}+\frac{e}{2} \mathbf{B}_{0} \times \mathbf{r}=\mathbf{P}_{\text {kin }}+e \mathbf{B}_{0} \times \mathbf{r}$ is a conserved quantity when time-evolution is governed by $H_{0}$, as in the classical theory. The relativistic correction $H_{\text {rel }}$ will be added later. The transverse degrees of freedom of the electromagnetic quantum vacuum are described by the Hamiltonian

$$
H_{F}=\sum_{\mathbf{k} \epsilon} \hbar \omega_{k}\left[a_{\mathbf{k} \epsilon}^{\dagger} a_{\mathbf{k} \epsilon}+\frac{1}{2}\right]
$$

The interaction with the quantum vacuum, when transformed reads [1,

$$
\begin{array}{r}
\tilde{W}=-\frac{e}{m_{1}}\left(\mathbf{p}+\frac{m_{1}}{M} \mathbf{P}-\frac{e}{2} \mathbf{B}_{0} \times \mathbf{r}\right) \cdot \mathbf{A}\left(\mathbf{R}+\frac{m_{2}}{M} \mathbf{r}\right) \\
-\frac{e}{m_{2}}\left(\mathbf{p}-\frac{m_{2}}{M} \mathbf{P}+\frac{e}{2} \mathbf{B}_{0} \times \mathbf{r}\right) \cdot \mathbf{A}\left(\mathbf{R}-\frac{m_{1}}{M} \mathbf{r}\right)
\end{array}
$$

$$
+\frac{e^{2}}{2 m_{1}} \mathbf{A}^{2}\left(\mathbf{R}+\frac{m_{2}}{M} \mathbf{r}\right)+\frac{e^{2}}{2 m_{2}} \mathbf{A}^{2}\left(\mathbf{R}-\frac{m_{1}}{M} \mathbf{r}\right)
$$

where the vector potential of the electromagnetic field is given by

$$
\mathbf{A}(\mathbf{x})=\sum_{\mathbf{k} \epsilon} \mathcal{A}_{\mathbf{k}} \hat{\epsilon}\left[a_{\mathbf{k} \epsilon} \exp (i \mathbf{k} \cdot \mathbf{x})+a_{\mathbf{k} \epsilon}^{\dagger} \exp (-i \mathbf{k} \cdot \mathbf{x})\right]
$$

with $\mathcal{A}_{k}=\sqrt{\hbar / 2 \varepsilon_{0} c_{0} k V}$ in a quantization volume $V$. In the presence of the quantum vacuum the pseudo momentum that commutes with $\tilde{H}$ is

$$
\tilde{\mathbf{K}}=\mathbf{P}+e \Delta \mathbf{A}+\sum_{\mathbf{k} \epsilon} \hbar \mathbf{k} a_{\mathbf{k} \epsilon}^{\dagger} a_{\mathbf{k} \epsilon}
$$

Because $\mathbf{K}$ is a conserved quantity, the time variation of $\mathbf{P}_{\text {kin }}$, and thus the total exerted force, equals minus the time-derivative of all other contributions to $\mathbf{K}$. If $\left|\Psi_{0}\right\rangle$ is the ground state of the joint atom and photon field, with $\left|\tilde{\Psi}_{0}\right\rangle=U^{*}\left|\Psi_{0}\right\rangle$ the transformed ground state, the pseudomomentum follows from,

$$
\begin{aligned}
\langle\mathbf{K}\rangle= & \left\langle\tilde{\Psi}_{0}|\tilde{\mathbf{K}}| \tilde{\Psi}_{0}\right\rangle \\
= & \left\langle\tilde{\Psi}_{0}\left|\tilde{\mathbf{P}}_{\text {kin }}\right| \tilde{\Psi}_{0}\right\rangle+e^{2} \mathbf{B}_{0} \times\left\langle\tilde{\Psi}_{0}|\mathbf{r}| \tilde{\Psi}_{0}\right\rangle \\
& +e\left\langle\tilde{\Psi}_{0}|\Delta \mathbf{A}| \tilde{\Psi}_{0}\right\rangle+\left\langle\tilde{\Psi}_{0}\left|\sum_{\mathbf{k} \epsilon} \hbar \mathbf{k} a_{\mathbf{k} \epsilon}^{\dagger} a_{\mathbf{k} \epsilon}\right| \tilde{\Psi}_{0}\right\rangle \\
\equiv & \left\langle\mathbf{P}_{\text {kin }}\right\rangle+\mathbf{P}_{\mathrm{A}}+\delta\left\langle\mathbf{P}_{\text {pola }}\right\rangle+\left\langle\mathbf{P}_{\text {long }}\right\rangle+\left\langle\mathbf{P}_{\text {trans }}\right\rangle
\end{aligned}
$$

The last two terms are the "longitudinal" and "transverse" contributions to the momentum stemming from the electromagnetic quantum vacuum 18. The classical Abraham momentum $\mathbf{P}_{\mathrm{A}}$ is found from the second term in on the righthand side when we ignore the coupling with the quantum vacuum. In this case $-e \mathbf{E}_{\mathbf{0}} \cdot \mathbf{r}$ becomes the only perturbing potential and we find,

$$
\begin{aligned}
\left|\tilde{\Psi}_{0}(W=0)\right\rangle & =\left[|0\rangle+\sum_{j \neq 0}|j\rangle \frac{\left\langle j\left|e \mathbf{E}_{\mathbf{0}} \cdot \mathbf{r}\right| 0\right\rangle}{E_{j}-E_{0}}\right] \otimes|\{0\}\rangle \\
& \equiv\left|0, \mathbf{E}_{0}\right\rangle \otimes|\{0\}\rangle
\end{aligned}
$$


where $|\{0\}\rangle$ denotes the quantum vacuum, empty for all plane waves, and $|j\rangle$ represent the unperturbed eigen states of the atom. We will denote by $\left|0, \mathbf{E}_{0}\right\rangle$ the atomic ground state with explicit reference to the the external electric field, and given by the first factor in the above expression. Insertion directly generates $\mathbf{P}_{\mathrm{A}}=\varepsilon_{0} \alpha(0) \mathbf{B}_{0} \times \mathbf{E}_{0}$ with the well-known expression for the static polarizability,

$$
\alpha(0)=\frac{2}{3} \frac{e^{2}}{\varepsilon_{0}} \sum_{j \neq 0} \frac{|\langle j|\mathbf{r}| 0\rangle|^{2}}{E_{j}-E_{0}}
$$

which for the hydrogen atom equals $\alpha(0)=18 \pi a_{0}^{3}[19$.

To find the contribution of the quantum vacuum to $\mathbf{K}$, we need to know how the ground state $\left|i=0, \mathbf{Q}_{0},\{n=0\}\right\rangle$ of an atom with pseudo-momentum $\mathbf{Q}_{0}$ in an empty vacuum is perturbed by the coupling $W$. Let $|j \mathbf{Q}\{n\}\rangle$ be an eigen state of $\tilde{H}_{0}, \mathbf{P}$ and $\tilde{H}_{F}$, the second with eigenvalue Q. We will restrict ourself to processes involving absorption and creation of one photon, i.e. involving the products of only two $\mathbf{A}(\mathbf{r})$ photon fields. The ground state is, up to second order in the coupling, equal to (the accent ' is to avoid summing over ground state itself),

$$
\begin{aligned}
\left|\tilde{\Psi}_{0}\right\rangle & =\left[1-\frac{1}{2} \sum_{i \mathbf{Q} n}^{\prime} \frac{\left|W_{i \mathbf{Q} n, 0 \mathbf{Q}_{0} 0}\right|^{2}}{\left(E_{0 \mathbf{Q}_{0} 0}-E_{i \mathbf{Q} n}\right)^{2}}\right]\left|0 \mathbf{Q}_{0}\{0\}\right\rangle \\
& +\sum_{i \mathbf{Q} n}^{\prime} \frac{W_{i \mathbf{Q} n, 0 \mathbf{Q}_{0} 0}}{E_{0 \mathbf{Q}_{0} 0}-E_{i \mathbf{Q} n}}|i \mathbf{Q} n\rangle \\
& +\sum_{i \mathbf{Q} n}^{\prime} \sum_{i^{\prime} \mathbf{Q}^{\prime} n^{\prime}}^{\prime} \frac{W_{i \mathbf{Q} n, i^{\prime} \mathbf{Q}^{\prime} n^{\prime}} W_{i^{\prime} \mathbf{Q}^{\prime} n^{\prime}, 0 \mathbf{Q}_{0} 0}}{\left(E_{0 \mathbf{Q}_{0} 0}-E_{i \mathbf{Q} n}\right)\left(E_{0 \mathbf{Q}_{0} 0}-E_{i^{\prime} \mathbf{Q}^{\prime} n^{\prime}}\right)}|i \mathbf{Q} n\rangle \\
& \left.-W_{0 \mathbf{Q}_{0} 0,0 \mathbf{Q}_{0} 0} \sum_{i \mathbf{Q} n}^{\prime} \frac{W_{i \mathbf{Q} n, 0 \mathbf{Q}_{0} 0}}{\left(E_{0 \mathbf{Q}_{0} 0}-E_{i \mathbf{Q} n}\right)^{2}}\right\rangle|i \mathbf{Q} n\rangle
\end{aligned}
$$

Note that the $\mathbf{A}^{2}(\mathbf{r})$ terms contained in $W$ do not contribute in this expression since $\left\langle i \neq 0\left|\mathbf{A}^{2}(\mathbf{r})\right| 0\right\rangle=0$ for any atomic state other than the ground state. Note also that the last term in the perturbed wave function does not contain one-photon processes since $\langle\{0\}|\mathbf{A}(\mathbf{r})|\{0\}\rangle=0$. Several terms yield diverging contributions to the momentum $\mathbf{K}$ and will below be identified. The factor in the first term proportional to the unperturbed ground state is imposed by normalization. In Appendix A we estimate it to be of order $\alpha^{3}$, which makes it beyond the scope of this work.

\section{$3.1 \delta\left\langle\mathbf{P}_{\text {pola }}\right\rangle$}

The momentum $\delta\left\langle\mathbf{P}_{\text {pola }}\right\rangle$ can be regarded as a quantum vacuum contribution to the static polarizability at $\mathbf{B}_{0}=0$ that finds its way to the Abraham momentum via the classical expression. As such it is arguably not a true "Casimir" momentum. It takes the form,

$$
\begin{aligned}
& \delta\left\langle\mathbf{P}_{\text {pola }}\right\rangle=e \mathbf{B}_{0} \times\left\langle 0, \mathbf{Q}_{0}, \mathbf{E}_{0}\{0\}\right| \mathbf{r} \sum_{i \mathbf{Q} n}^{\prime} \sum_{i^{\prime} \mathbf{Q}^{\prime} n^{\prime}}^{\prime} \\
& \frac{W_{i \mathbf{Q} n, i^{\prime} \mathbf{Q}^{\prime} n^{\prime}} W_{i^{\prime} \mathbf{Q}^{\prime} n^{\prime}, 0 \mathbf{Q}_{0} 0}}{\left(E_{0 \mathbf{Q}_{0} 0}-E_{i \mathbf{Q} n}\right)\left(E_{0 \mathbf{Q}_{0} 0}-E_{i^{\prime} \mathbf{Q}^{\prime} n^{\prime}}\right)}|i \mathbf{Q} n\rangle+c . c .
\end{aligned}
$$

The matrix elements of $W$ contain exponentials of the form $\exp \left( \pm i \mathbf{k} \cdot \mathbf{r}_{i}\right)$ stemming from $\mathbf{A}(\mathbf{r})$. Those terms for which products occur of exponentials with opposing phase, diverge. They can be re-arranged to give,

$$
\begin{aligned}
& \delta\left\langle\mathbf{P}_{\text {pola }}\right\rangle_{\text {div }}=2 \operatorname{Re} \frac{e^{2}}{m_{1}^{2}} \sum_{\mathbf{k} \epsilon} \mathcal{A}_{k}^{2} \\
& \left\langle 0, \mathbf{E}_{0}\right| e\left(\mathbf{B}_{0} \times \mathbf{r}\right) \frac{1^{\prime}}{\tilde{H}_{0}\left(\mathbf{p}, \mathbf{Q}_{0}\right)-E_{0 \mathbf{Q}_{0} 0}}\left(\mathbf{p}+\frac{m_{1}}{M} \mathbf{Q}_{0}\right) \cdot \epsilon \\
& \frac{1}{\tilde{H}_{0}\left(\mathbf{p}+\frac{m_{2}}{M} \hbar \mathbf{k}, \mathbf{Q}_{0}-\hbar \mathbf{k}\right)-E_{0 \mathbf{Q}_{0} 0}+\hbar \omega_{k}}\left(\mathbf{p}+\frac{m_{1}}{M} \mathbf{Q}_{0}\right) \cdot \epsilon\left|0, \mathbf{E}_{0}\right\rangle
\end{aligned}
$$

plus a similar term for $m_{2}$. The extra momentum and energy in denominator of the second line stem from the 
absorption of a virtual photon in the intermediate photon state $\{n\}$. This changes the total state and thus removes the accent in the sum over the remaining atomic states. It generates a term $\hbar^{2} k^{2} / 2 m_{1(2)}+\hbar \omega_{k}$ and leads to a $k$-integral that diverges in the UV. Upon extracting the divergency leads to,

$$
\begin{aligned}
& \delta\left\langle\mathbf{P}_{\text {pola }}\right\rangle_{\text {div }}= \\
& \frac{1}{2} \frac{\delta m_{1}}{m_{1}^{2}}\left\langle 0, \mathbf{E}_{0}\left|e\left(\mathbf{B}_{0} \times \mathbf{r}\right) \frac{1^{\prime}}{\tilde{H}_{0}-E_{0 \mathbf{Q}_{0} 0}}\left(\mathbf{p}+\frac{m_{1}}{M} \mathbf{Q}_{0}\right)^{2}\right| 0, \mathbf{E}_{0}\right\rangle \\
& +\frac{1}{2} \frac{\delta m_{2}}{m_{2}^{2}}\left\langle 0\left|e\left(\mathbf{B}_{0} \times \mathbf{r}\right) \frac{1^{\prime}}{\tilde{H}_{0}-E_{0 \mathbf{Q}_{0} 0}}\left(\mathbf{p}-\frac{m_{2}}{M} \mathbf{Q}_{0}\right)^{2}\right| 0, \mathbf{E}_{0}\right\rangle \\
& +c . c
\end{aligned}
$$

where we have introduced the masses,

$$
\delta m_{i}=\frac{4}{3 \pi} \alpha \hbar^{2} \int_{0}^{\infty} \frac{d k k}{\hbar^{2} k^{2} / 2 m_{i}+\hbar \omega_{k}}
$$

The diverging mass is recognized as the nonrelativistic QED contribution to the bare mass [14, and is supposed to be absorbed by the value attributed to the observed mass. This "Kramer-Bethe" mass renormalization method seems reasonable provided that all masses acquire the same diverging contribution. The polarizability is in principle a complicated function of the reduced mass. It is easy to check that $\delta(1 / \mu)=-\delta m_{1} / m_{1}^{2}-\delta m_{2} / m_{2}^{2}$. As a result, the $\mathbf{p}^{2}$ term in the expression for $\delta\left\langle\mathbf{P}_{\text {pola }}\right\rangle_{\text {div }}$ contributes

$$
\begin{aligned}
& \delta\left\langle\mathbf{P}_{\text {pola }}\right\rangle_{\text {div }}= \\
& e \mathbf{B}_{0} \times\left\langle 0, \mathbf{E}_{0}\left|\mathbf{r} \frac{-1^{\prime}}{\tilde{H}_{0}-E_{0}} \delta(1 / 2 \mu) \mathbf{p}^{2}\right| 0, \mathbf{E}_{0}\right\rangle+c . c \\
& =e \mathbf{B}_{0} \times\left(\left\langle 0, \mathbf{E}_{0}|\mathbf{r}| \delta 0, \mathbf{E}_{0}\right\rangle+c . c .\right) \\
& =\varepsilon_{0} \delta \alpha(0) \mathbf{B}_{0} \times \mathbf{E}_{0}
\end{aligned}
$$

where $\left|\delta 0, \mathbf{E}_{0}\right\rangle=-\left(\tilde{H}_{0}-E_{0}\right)^{\prime-1} \delta \tilde{V}\left|0, \mathbf{E}_{0}\right\rangle$ is the first-order modification of the atomic ground state due to the per- turbation $\delta \tilde{V}=\delta(1 / 2 \mu) \mathbf{p}^{2}$ in the kinetic energy. We thus conclude that this divergence disappears when all masses in $\alpha(0)$ absorb consistently the same diverging QED contribution (14).

The terms proportional to $\mathbf{Q}_{0}^{2}$ and $\mathbf{p} \cdot \mathbf{Q}_{0}$ in $\delta\left\langle\mathbf{P}_{\text {pola }}\right\rangle_{\text {div }}$ can be seen to vanish. The other terms in $\delta\left\langle\mathbf{P}_{\text {pola }}\right\rangle$ are finite. A straightforward analysis comparable to the one for $\delta\left\langle\mathbf{P}_{\text {long }}\right\rangle$ below shows it be a factor $\alpha^{2} m_{e} / M$ smaller than $\mathbf{P}_{A}$. In this work we shall restrict ourselves to orders $\alpha^{2}$. We conclude that no quantum vacuum contribution of the order $\alpha^{2}$ exists to the static polarizability.

\section{$3.2\left\langle\mathbf{P}_{\text {long }}\right\rangle$}

The quantum expectation of the momentum of the longitudinal vacuum field reads,

$$
\begin{aligned}
\left\langle\mathbf{P}_{\text {long }}\right\rangle= & \left\langle 0, \mathbf{E}_{0}, \mathbf{Q}_{0},\{0\}\right|\left(e \Delta \mathbf{A}\left(\mathbf{r}_{1}\right)-e \Delta \mathbf{A}\left(\mathbf{r}_{2}\right)\right) \times \\
& \sum_{i \mathbf{Q} n}^{\prime} \mathcal{A}_{k}^{2} \frac{W_{i \mathbf{Q} n, 0 \mathbf{Q}_{0} 0}}{\left(E_{0 \mathbf{Q}_{0} 0}-E_{i \mathbf{Q} n}\right)}|i \mathbf{Q} n\rangle+c . c .
\end{aligned}
$$

If we acknowledge the subsequent annihilation and creation of one virtual photon, we find that

$$
\begin{aligned}
&\left\langle\mathbf{P}_{\text {long }}\right\rangle=2 e^{2} \operatorname{Re} \sum_{\mathbf{k} \epsilon} \mathcal{A}_{k}^{2} \\
&\left\{\left\langle 0, \mathbf{E}_{0}\right|\left(1-e^{-i \mathbf{k} \cdot \mathbf{r}}\right) \times\right. \\
& \frac{1}{\tilde{H}_{0}\left(\mathbf{p}-\frac{m_{2}}{M} \hbar \mathbf{k}, \mathbf{Q}_{0}-\hbar \mathbf{k}\right)-E_{0} \mathbf{Q}_{0} 0}+\hbar \omega_{k} \\
& \epsilon \cdot\left(\frac{\mathbf{p}}{m_{1}}+\frac{\mathbf{Q}_{0}}{M}-\frac{e}{2 m_{1}} \mathbf{B}_{0} \times \mathbf{r}\right)\left|0, \mathbf{E}_{0}\right\rangle \\
&+\left\langle 0, \mathbf{E}_{0}\right|\left(e^{i \mathbf{k} \cdot \mathbf{r}}-1\right) \times \\
& \frac{1}{\tilde{H}_{0}\left(\mathbf{p}+\frac{m_{1}}{M} \hbar \mathbf{k}, \mathbf{Q}_{0}-\hbar \mathbf{k}\right)-E_{0} \mathbf{Q}_{0} 0}+\hbar \omega_{k} \\
&\left.\epsilon \cdot\left(\frac{\mathbf{p}}{m_{2}}-\frac{\mathbf{Q}_{0}}{M}+\frac{e}{2 m_{2}} \mathbf{B}_{0} \times \mathbf{r}\right)\left|0, \mathbf{E}_{0}\right\rangle\right\}
\end{aligned}
$$


It is clear that terms involving exponents $\exp ( \pm i \mathbf{k} \cdot \mathbf{r})$ ensure finite $k$-integrals. We will first focuss on the diverging terms. We can extract the divergence by keeping only recoil plus photon energy in the denominator, to get

$$
\begin{aligned}
\left\langle\mathbf{P}_{\text {long }}\right\rangle_{\text {div }} & =\delta m_{1}\left\langle 0, \mathbf{E}_{0}\left|\left(\frac{\mathbf{p}}{m_{1}}+\frac{\mathbf{Q}_{0}}{M}-\frac{e}{2 m_{1}} \mathbf{B}_{0} \times \mathbf{r}\right)\right| 0, \mathbf{E}_{0}\right\rangle \\
& +\delta m_{2}\left\langle 0, \mathbf{E}_{0}\left|\left(-\frac{\mathbf{p}}{m_{2}}+\frac{\mathbf{Q}_{0}}{M}-\frac{e}{2 m_{2}} \mathbf{B}_{0} \times \mathbf{r}\right)\right| 0, \mathbf{E}_{0}\right\rangle
\end{aligned}
$$

with $\boldsymbol{\Delta}_{\mathbf{k}}=\sum_{\epsilon_{k}} \epsilon_{k} \epsilon_{k}$ the transverse projection matrix.

The integral over $\mathbf{k}$ can be carried out by using that $\int_{0}^{\infty} d k \int d \Omega \exp (i \mathbf{k} \cdot \mathbf{r}) \boldsymbol{\Delta}_{\mathbf{k}}=\pi^{2}(1+\hat{\mathbf{r}} \hat{\mathbf{r}}) / r$ and we get, with the masses $\delta m_{i}$ defined earlier in Eq. (14). From the relation $\left[\mathbf{r}, \tilde{H}_{0}\right]=i \hbar \partial_{\mathbf{p}} \tilde{H}_{0}$ it is easy to show that $\left\langle 0, \mathbf{E}_{0}\right| \mathbf{p}+$ $(\Delta m / M)(e / 2) \mathbf{B}_{0} \times \mathbf{r}\left|0, \mathbf{E}_{0}\right\rangle=0$. This leads to

$$
\begin{aligned}
\left\langle\mathbf{P}_{\text {long }}\right\rangle_{\text {div }} & =\left(\mathbf{Q}_{0}-\frac{e}{2}\left\langle 0, \mathbf{E}_{0}\left|\mathbf{B}_{0} \times \mathbf{r}\right| 0, \mathbf{E}_{0}\right\rangle\right) \frac{\delta m_{1}+\delta m_{2}}{M} \\
& =\left\langle 0, \mathbf{E}_{0}\left|\tilde{\mathbf{P}}_{\text {kin }}\right| 0, \mathbf{E}_{0}\right\rangle \frac{\delta M}{M}=\left\langle\mathbf{P}_{\text {kin }}\right\rangle \frac{\delta M}{M}
\end{aligned}
$$

Hence, the diverging contribution disappears entirely into the inertial mass featuring in the kinetic momentum $\mathbf{P}_{\text {kin }}=$ $M \mathbf{v}$ of the atom.

We proceed with the converging terms. When we remove the divergencies from the expression even the part with compensating phase factors will yield a finite contribution. This can be shown to be of order $\alpha^{3} \mathbf{P}_{A}$ and will be ignored here. The terms with exponentials typically have $k \approx 1 / a_{0}$ since the ground state has $r \approx a_{0}$. In that case $\hbar \omega_{k} \gg e^{2} / 4 \pi \varepsilon_{0} a_{0}$ and the atomic energy $H_{0}$ can be neglected. Similarly the recoil energy $\hbar^{2} k^{2} / 2 m_{i}$ is much smaller than $\hbar \omega_{k}$. This simplification leads to the following expression,

$$
\begin{aligned}
& \left\langle\mathbf{P}_{\text {long }}\right\rangle= \\
& 2 e^{2} \sum_{\mathbf{k}} \frac{\mathcal{A}_{k}^{2}}{\hbar \omega_{k}}\left\{\left\langle 0, \mathbf{E}_{0}\right| \boldsymbol{\Delta}_{\mathbf{k}} \cdot \mathbf{p}\left(\frac{1}{m_{2}} e^{i \mathbf{k} \cdot \mathbf{r}}-\frac{1}{m_{1}} e^{-i \mathbf{k} \cdot \mathbf{r}}\right)\right. \\
& -\boldsymbol{\Delta}_{\mathbf{k}} \cdot \mathbf{Q}_{0} \frac{1}{M}\left(e^{i \mathbf{k} \cdot \mathbf{r}}+e^{-i \mathbf{k} \cdot \mathbf{r}}\right) \\
& \left.+\frac{e}{2} \boldsymbol{\Delta}_{\mathbf{k}} \cdot\left(\mathbf{B}_{0} \times \mathbf{r}\right)\left(\frac{1}{m_{2}} e^{i \mathbf{k} \cdot \mathbf{r}}+\frac{1}{m_{1}} e^{-i \mathbf{k} \cdot \mathbf{r}}\right)\right\}\left|0, \mathbf{E}_{0}\right\rangle(17)
\end{aligned}
$$

$$
\begin{aligned}
& \left\langle\mathbf{P}_{\text {long }}\right\rangle=\frac{e^{2}}{8 \pi \varepsilon_{0} c_{0}^{2}}\left\langle 0, \mathbf{E}_{0}\right| \frac{1+\hat{\mathbf{r}} \hat{\mathbf{r}}}{r} \\
& {\left[\mathbf{p}\left(\frac{1}{m_{2}}-\frac{1}{m_{1}}\right)-2 \frac{\mathbf{Q}_{0}}{M}+\frac{e}{2 \mu} \mathbf{B}_{0} \times \mathbf{r}\right]\left|0, \mathbf{E}_{0}\right\rangle}
\end{aligned}
$$

Since $\left\langle 0, \mathbf{Q}_{0}, \mathbf{E}_{0}\left|-e^{2} / 4 \pi \varepsilon_{0} r\right| 0, \mathbf{Q}_{0}, \mathbf{E}_{0}\right\rangle=2 E_{0}+\mathcal{O}\left(\mathbf{E}_{0}^{2}\right)$, the middle term generates a contribution,

$$
\left\langle\mathbf{P}_{\text {long }}\right\rangle_{2}=\frac{8}{3} \frac{E_{0}}{M c_{0}^{2}} \mathbf{Q}_{0}
$$

This can, in fact, be recognized as the contribution to the ground state energy of the modified Coulomb field seen by the moving charges, as expressed by the Darwin interaction [22], and thus, via the relativistic equivalence, to the inertial mass. The first term in the expression for $\left\langle\mathbf{P}_{\text {long }}\right\rangle$ is more elaborate. We can insert the expression (10) for the polarized ground state $\left|0, \mathbf{E}_{0}\right\rangle$, and expand $\tilde{H}_{0}$ linearly into the external magnetic field. Some algebra leads to the expression

$$
\begin{aligned}
& \left\langle\mathbf{P}_{\text {long }}\right\rangle_{1}=-\frac{e^{4}}{16 \pi \varepsilon_{0} c_{0}^{2}} \frac{\left(m_{2}-m_{1}\right)^{2}}{m_{1}^{2} m_{2}^{2}} \epsilon_{n m l} B_{n} E_{k} \\
& \left\langle 0\left|\mathbf{p} \cdot \frac{1+\hat{\mathbf{r}} \hat{\mathbf{r}}}{r} \frac{1^{\prime}}{\left(\tilde{H}_{0}-E_{0}\right)^{2}} r_{m} p_{l} r_{k}\right| 0\right\rangle+\text { c.c }
\end{aligned}
$$

This can be further simplified for the hydrogen isotropic $1 \mathrm{~S}$ ground state for which $p_{i}|0\rangle=\left(i \hbar / a_{0}\right) \hat{r}_{i}|0\rangle$, and using that $m_{1} \gg m_{2}=m_{e}$ so that

$$
\left\langle\mathbf{P}_{\text {long }}\right\rangle_{1}=-\mathbf{B}_{0} \times \mathbf{E}_{0} \frac{e^{4} \hbar^{2}}{4 \pi \varepsilon_{0} c_{0}^{2} m_{e}^{2} a_{0}} \frac{1}{3}\left\langle 0\left|\frac{1}{r} \hat{r}_{m} \frac{1^{\prime}}{\left(\tilde{H}_{0}-E_{0}\right)^{2}} r_{m}\right| 0\right\rangle
$$

If we write $\alpha(0)=18 \pi a_{0}^{3}$, this becomes

$$
\left\langle\mathbf{P}_{\text {long }}\right\rangle_{1}=-\kappa_{1} \alpha^{2} \mathbf{P}_{\mathrm{A}}
$$


with $\mathbf{P}_{\mathrm{A}}=\varepsilon_{0} \alpha(0) \mathbf{B}_{0} \times \mathbf{E}_{0}$ and the dimensionless number

$$
\kappa_{1}=\frac{2}{27}\left(\frac{e^{2}}{4 \pi \varepsilon_{0} a_{0}}\right)^{2} \sum_{n \neq 0 \ell m} \frac{\left\langle 0\left|r^{-1} \hat{\mathbf{r}}\right| n \ell m\right\rangle \cdot\langle n \ell m|\mathbf{r}| 0\rangle}{\left(E_{n}-E_{0}\right)^{2}}
$$

Since $\kappa_{1}>0$, this contribution lowers the classical Abraham momentum. The sum over all excited states (with $\ell=1$ imposed by selection rule, the sum over $m$ equals 1 ) involves both the bound states and the continuous spectrum. The sum over discrete states can easily be done numerically using a recursion formula for the hypergeometric functions 23]. With $E_{n}=-e^{2} / 8 \pi \varepsilon_{0} a_{0} n^{2}$ the numerical factor equals $\kappa_{2}(D)=0.21$. The part associated with continuous spectrum is much harder and we shall here assume that the continuous spectrum consists of plane waves $\exp (i \mathbf{q} \cdot \mathbf{r}) / \sqrt{V}$ with energy $E_{\mathbf{q}}=\hbar^{2} q^{2} / 2 m_{e}$, which is a usual approximation in treatises of the photo-electric effect 24] and valid in principle only for $q>1 / a_{0}$. This leads to $\left(y=q a_{0}\right)$,

$$
\begin{aligned}
& \kappa_{1}(C)=\frac{8}{27} \sum_{\mathbf{q}} \frac{\left\langle 0\left|r^{-1} \hat{\mathbf{r}}\right| \mathbf{q}\right\rangle \cdot\langle\mathbf{q}|\mathbf{r}| 0\rangle}{\left(a_{0}^{2} q^{2}+1\right)^{2}}= \\
& \frac{8}{27} \frac{16}{\pi} \int_{y_{\text {min }}}^{\infty} d y \frac{y^{3}}{\left(y^{2}+1\right)^{3}}\left(\frac{\arctan y}{y^{2}}-\frac{1}{y \sqrt{y^{2}+1}}\right)(24)
\end{aligned}
$$

Choosing $y_{\min }=0$ or $y_{\min }=1$ as lower limits gives 9.3 . $10^{-3}<\kappa_{1}(C)<1.4 \cdot 10^{-2}$. This is small compared to $\kappa_{1}(D)$. We shall adopt $\kappa_{1}=0.22$ [25].

We finally evaluate the last term in Eq. (18). Upon inserting the ground state (10) perturbed by the electric field. this leads to

$$
\left\langle\mathbf{P}_{\text {long }}\right\rangle_{2}=\frac{e^{2}}{4 \pi \varepsilon_{0}} \frac{e^{2}}{6 m_{e} c_{0}^{2}} \mathbf{B}_{0} \times \mathbf{E}_{0} \sum_{j}^{\prime} \frac{\langle 0|\hat{\mathbf{r}}| j\rangle \cdot\langle j|\mathbf{r}| 0\rangle}{E_{j}-E_{0}}
$$

We can write this as

$$
\left\langle\mathbf{P}_{\text {long }}\right\rangle_{2}=+\kappa_{2} \alpha^{2} \mathbf{P}_{\mathrm{A}}
$$

with the dimensionless number

$$
\kappa_{2}=\frac{1}{27} \frac{e^{2}}{4 \pi \varepsilon_{0} a_{0}^{2}} \sum_{n \neq 0 \ell=1 m} \frac{\langle 0|\hat{\mathbf{r}}| n \ell m\rangle \cdot\langle n \ell m|\mathbf{r}| 0\rangle}{E_{n}-E_{0}}
$$

The same method as above yields $\kappa_{2}(D)=0.0796$ for the discrete spectrum. For the continuous spectrum we find, again assuming perfect plane waves,

$$
\kappa_{2}(C)=\frac{256}{27 \pi} \int_{y_{\min }}^{\infty} d y \frac{y^{4}}{\left(y^{2}+1\right)^{6}} \approx 0.018
$$

assuming a lower bound at $y=q a_{0}=1$. Thus $\kappa_{2} \approx 0.1$.

We see that $\left\langle\mathbf{P}_{\text {long }}\right\rangle_{1}$ en $\left\langle\mathbf{P}_{\text {long }}\right\rangle_{2}$ have opposite sign. We conclude that $\left\langle\mathbf{P}_{\text {long }}\right\rangle \approx-0.12 \alpha^{2} \mathbf{P}_{A}$

\section{$3.3\left\langle\mathbf{P}_{\text {trans }}\right\rangle$}

The leading contribution to the quantum expectation value of the transverse momentum of electromagnetic field is given by,

$$
\begin{aligned}
\left\langle\mathbf{P}_{\text {trans }}\right\rangle= & e^{2} \sum_{\mathbf{k} \epsilon} \mathcal{A}_{k}^{2} \hbar \mathbf{k} \times \\
& \left\langle 0, \mathbf{E}_{0}\right| \epsilon \cdot\left(\frac{\mathbf{p}}{m_{e}}-\frac{\mathbf{Q}_{0}}{M}+\frac{e}{2 m_{e}} \mathbf{B}_{0} \times \mathbf{r}\right) \\
& \frac{1}{\left(\tilde{H}_{0}\left(\mathbf{p}+\hbar \mathbf{k}, \mathbf{Q}_{0}-\hbar \mathbf{k}\right)-E_{0} \mathbf{Q}_{0} 0+\hbar \omega_{k}\right)^{2}} \\
& \epsilon \cdot\left(\frac{\mathbf{p}}{m_{e}}-\frac{\mathbf{Q}_{0}}{M}+\frac{e}{2 m_{e}} \mathbf{B}_{0} \times \mathbf{r}\right)\left|0, \mathbf{E}_{0}\right\rangle
\end{aligned}
$$

and a similar term for the proton (particle 1), which in fact can be neglected for $m_{2}=m_{e} \ll m_{1}$. In leading order, atomic energies can be neglected compared to typical photon energies $\hbar \omega_{k}$. We need to expand the denominator 
into $\mathbf{k}$ in order to find a non-vanishing contribution. This leads to,

$$
\begin{aligned}
\left\langle\mathbf{P}_{\text {trans }}\right\rangle= & \frac{e^{2} \hbar}{2 \varepsilon_{0} c_{0}} \int \frac{d^{3} \mathbf{k}}{(2 \pi)^{3}} \frac{2 \hbar \mathbf{k}}{k\left(\hbar^{2} k^{2} / 2 m_{e}+\hbar \omega_{k}\right)^{3}} \\
& \left\langle 0, \mathbf{E}_{0}\right|\left(\frac{\mathbf{p}}{m_{e}}-\frac{\mathbf{Q}_{0}}{M}+\frac{e}{2 m_{e}} \mathbf{B}_{0} \times \mathbf{r}\right) .
\end{aligned}
$$$$
\boldsymbol{\Delta}_{\mathbf{k}}\left[\frac{1}{m_{e}} \hbar \mathbf{k} \cdot \frac{e}{2}\left(\mathbf{B}_{0} \times \mathbf{r}\right)+\frac{1}{m_{e}} \hbar \mathbf{k} \cdot \mathbf{p}+\frac{1}{M} \hbar \mathbf{k} \cdot \mathbf{Q}_{0}\right.
$$$$
\cdot\left(\frac{\mathbf{p}}{m_{e}}-\frac{\mathbf{Q}_{0}}{M}+\frac{e}{2 m_{e}} \mathbf{B}_{0} \times \mathbf{r}\right)\left|0, \mathbf{E}_{0}\right\rangle
$$

Since $\int_{0}^{\infty} d k k^{3} /\left(\hbar^{2} k^{2} / 2 m_{e}+\hbar \omega_{k}\right)^{3}=m_{e} / \hbar^{4} c_{0}^{2}$ this can be evaluated. For instance, the typical contribution linear to $\mathrm{Q}_{0}$ will be,

$$
\begin{aligned}
\left\langle\mathbf{P}_{\text {trans }}\right\rangle & \simeq \frac{e^{2}}{4 \pi \varepsilon_{0}} \frac{1}{\pi \hbar c_{0}^{3} m_{e}}\left\langle 0\left|\mathbf{p}^{2}\right| 0\right\rangle \frac{\mathbf{Q}_{0}}{M} \\
& \simeq \frac{e^{2}}{4 \pi \varepsilon_{0}} \frac{1}{\pi \hbar c_{0}^{3} m_{e}} m_{e} E_{0} \frac{\mathbf{Q}_{0}}{M} \\
& \simeq \alpha \frac{E_{0}}{M c_{0}^{2}} \mathbf{Q}_{0}
\end{aligned}
$$

Similarly we can estimate that terms proportional to $\mathbf{B}_{0} \times$ $\mathbf{r}$ generate a momentum of order $\alpha^{3} \mathbf{P}_{\mathrm{A}}$. Thus, $\left\langle\mathbf{P}_{\text {trans }}\right\rangle$ is typically a factor $\alpha$ smaller than the longitudinal momentum $\left\langle\mathbf{P}_{\text {long }}\right\rangle$ found in Eqs. (19) and (22). It will be neglected in this work.

\section{Relativistic corrections to the}

\section{Pseudo-momentum}

A particle with mass $m$ and kinetic momentum $\mathbf{p}$ achieves a relativistic correction $-p^{4} / 8 c_{0}^{2} m^{3}$ to its kinetic energy. When exposed to external electromagnetic fields , the particles Hamiltonian achieves a term

$$
\begin{aligned}
H_{\text {rel }}= & -\frac{1}{8 c_{0}^{2} m_{1}^{3}}\left|\mathbf{p}+\frac{m_{1}}{M} \mathbf{P}-e \mathbf{A}_{1}\right|^{4} \\
& -\frac{1}{8 c_{0}^{2} m_{2}^{3}}\left|-\mathbf{p}+\frac{m_{2}}{M} \mathbf{P}+e \mathbf{A}_{2}\right|^{4}
\end{aligned}
$$

It is easily shown that $\tilde{H}_{\text {rel }}=U^{*} H_{\text {rel }} U$ commutes with the canonical total momentum $\mathbf{P}$. The kinetic momentum is related to the conjugate total momentum by the relation $\mathbf{P}_{\text {kin }}=M \dot{\mathbf{R}}=M \partial_{\mathbf{P}} H$. Equation (8) for the pseudomomentum is still valid but Eq. (9) achieves an extra term ${ }_{0} \mathbf{P}_{\text {rel }}=-M \partial_{\mathbf{P}} H_{\text {rel }}$. Straightforward algebra leads to

$$
\begin{aligned}
\tilde{\mathbf{P}}_{\text {rel }} & =\frac{1}{2 m_{1}^{2} c_{0}^{2}}\left(\mathbf{p}+\frac{m_{1}}{M} \mathbf{P}-\frac{e}{2} \mathbf{B}_{0} \times \mathbf{r}\right)^{3} \\
& +\frac{1}{2 m_{2}^{2} c_{0}^{2}}\left(-\mathbf{p}+\frac{m_{2}}{M} \mathbf{P}-\frac{e}{2} \mathbf{B}_{0} \times \mathbf{r}\right)^{3}
\end{aligned}
$$

with notation $\mathbf{w}^{3}:=(\mathbf{w} \cdot \mathbf{w}) \mathbf{w}$. In the following we collect different contributions to $\left\langle 0, \mathbf{E}_{0}\left|\tilde{\mathbf{P}}_{\text {rel }}\right| 0, \mathbf{E}_{0}\right\rangle$, that are either linear in $\mathbf{Q}_{0}$ or in $\mathbf{B}_{0} \times \mathbf{E}_{0}$.

The contributions linear to $\mathbf{P}$ add up to

$$
\begin{aligned}
\left\langle\mathbf{P}_{\text {rel }}\right\rangle_{1} & =\left\langle 0, \mathbf{E}_{0}\left|\frac{1}{2 M c_{0}^{2}} \frac{1}{\mu}\left[\mathbf{p}^{2} \mathbf{P}+2(\mathbf{p} \cdot \mathbf{P}) \mathbf{p}\right]\right| 0, \mathbf{E}_{0}\right\rangle \\
& =\frac{1}{M c_{0}^{2}} \frac{5}{3} \mathbf{Q}_{0}\left\langle 0, \mathbf{E}_{0}\left|\frac{\mathbf{p}^{2}}{2 \mu}\right| 0, \mathbf{E}_{0}\right\rangle \\
& =-\frac{5}{3} \frac{E_{0}}{M c_{0}^{2}} \mathbf{Q}_{0}
\end{aligned}
$$

If we next split off $\tilde{\mathbf{P}}_{\text {rel,1 }}$ from the equation and assume that $m_{1} \gg m_{2}=m_{e}$, we obtain,

$$
\tilde{\mathbf{P}}_{\mathrm{rel}}=\tilde{\mathbf{P}}_{\mathrm{rel}, 1}-\frac{1+\mathcal{O}\left(m_{e}^{2} / m_{p}^{2}\right)}{2 m_{e}^{2} c_{0}^{2}}\left(\mathbf{p}+\frac{e}{2} \mathbf{B}_{0} \times \mathbf{r}\right)^{3}
$$

In Appendix B we show that

$$
\left\langle 0, \mathbf{E}_{0}\left|\left(\mathbf{p}+\frac{e}{2} \frac{\Delta m}{M} \mathbf{B}_{0} \times \mathbf{r}\right)^{3}\right| 0, \mathbf{E}_{0}\right\rangle=0
$$

Using this identity and a little algebra leads to,

$$
\begin{aligned}
& \left\langle\mathbf{P}_{\text {rel }}\right\rangle=\left\langle\mathbf{P}_{\text {rel }}\right\rangle_{1}-\frac{m_{e}}{M} \frac{1}{2 m_{e}^{2} c_{0}^{2}} \times \\
& \left(e^{2}\left\langle 0\left|\left(\mathbf{E}_{0} \cdot \mathbf{r}\right) \frac{\left(\mathbf{B}_{0} \cdot \mathbf{L}\right)^{\prime}}{\tilde{H}_{0}-E_{0}} \mathbf{p}\right| 0\right\rangle+\frac{e}{2}\left\langle 0, \mathbf{E}_{0}\left|\mathbf{p}^{2} \mathbf{B}_{0} \times \mathbf{r}\right| 0, \mathbf{E}_{0}\right\rangle\right)
\end{aligned}
$$

with $\mathbf{L}=\mathbf{r} \times \mathbf{p}$. This expression can be evaluated for the hydrogen atom, but we will here restrict to an order of 
magnitude. It follows straightforwardly that $(\cdots) / m_{e}^{2} c_{0}^{2} \sim$ $\alpha^{2} \mathbf{P}_{\mathrm{A}}$. We thus conclude that

$$
\left\langle\mathbf{P}_{\text {rel }}\right\rangle=-\frac{5}{3} \frac{E_{0}}{M c_{0}^{2}} \mathbf{Q}_{0}+\mathcal{O}\left(\alpha^{2} \frac{m_{e}}{M}\right) \varepsilon_{0} \alpha(0) \mathbf{B}_{0} \times \mathbf{E}_{0}
$$

Both terms are of order $\alpha^{2} m_{e} / M$ and together with the QED contribution (19) the first adds up to the value $E_{0} / M c_{0}^{2}$ that we could have anticipated from the relativistic equivalence principle that includes the bounding energy $E_{0} / c_{0}^{2}$ into inertial mass. This finding fits into the general notion that Casimir energy has inertial mass that respects the equivalence principle [15]. The relativistic correction to the Abraham momentum is a factor $m_{e} / M$ smaller than the contribution from the quantum vacuum, found in Eq. (22).

There is also a modification of the static polarizability imposed by special relativity. For the hydrogen atom the relative correction was calculated by Bartlett and Power [21] to be $-\frac{28}{27} \alpha^{2}$. This will give a similar contribution to the Abraham momentum as the one stemming from the quantum vacuum. We will here take the point of view that the atom's relativistic correction to the Abraham momentum entering via the static polarizability is not counted as Casimir momentum, and adopt $\mathbf{P}_{A}$ with either the $o b$ served or the exactly calculated $\alpha(0)$ as the true Abraham momentum. This is also our experimental procedure [7.

\section{Conclusion}

In this work we have calculated the contribution of the quantum vacuum to the Abraham force. Our approach treats the kinetic momentum $M \mathbf{v}$ and the magneto-electric Abraham contribution on equal footing. The conserved pseudo-momentum is the sum of kinetic and Abraham momentum, $\mathbf{K}=\mathbf{K}_{\text {kin }}+\mathbf{P}_{\mathrm{A}}$, so that the force is $\partial_{t} \mathbf{K}_{\text {kin }}=$ $M \ddot{\mathbf{R}}=-\partial_{t} \mathbf{P}_{\mathrm{A}}$. As expected from the equivalence principle, our approach shows that the inertial mass $M$ is affected by the binding energy of the atom, with a significant contribution that can be viewed as stemming from the longitudinal electromagnetic field of the quantum vacuum, but which in electrodynamics is better known as the Darwin interaction associated with moving charges 22]. As for contribution of the quantum vacuum to the Abraham momentum, our main result is that, at least for the simple case of the hydrogen atom, divergencies can be uniquely renormalized into the masses of electron and proton. The finite small remainder is of relative order $-0.12 \alpha^{2} \pm \mathcal{O}\left(\alpha^{3}\right) \sim 6 \cdot 10^{-6}$, thus reducing the classical value, and stemming from the gauge potential $e \mathbf{A}$ of the quantum vacuum. The transverse virtual photons with momentum $\hbar \mathbf{k}$ contribute only to order $\alpha^{3}$. The static polarizability $\alpha(0)$ itself has a well-known relativistic correction of order $-\alpha^{2}$, whereas the contribution from the quantum vacuum to $\alpha(0)$ is much smaller, only of order $\alpha^{2} m_{e} / M$. We have corrected an error in a previous publication [1] where a modification of order $\alpha$ was predicted for the relative change in the Abraham momentum. In the present work, we have also considered relativistic corrections to the Abraham force and have concluded them to be factor $m_{e} / M=5 \cdot 10^{-4}$ smaller than the contribution of the quantum vacuum. 
The QED approach in this work, very likely to be valid albeit more complex for more complex atoms and molecules, solves the UV catastrophe encountered in a semi-classical approach 8]. Casimir momentum exists, is finite, but is of relative order $\alpha^{2}$. How such "Casimir momentum" reveals itself in more complex quantum systems is an important though difficult many-body problem. One could speculate it to scale like $(Z \alpha)^{2}$, with $Z$ the atomic number, as is known to be true for relativistic corrections to the static polarizability of hydrogen-like atoms [21]. If this is true the "Casimir momentum" could become within reach of experimental observation.

This work was supported by the ANR contract PHOTONIMPULS ANR-09-BLAN-0088-01. We would like to thank Denis Basko and Thierry Champel for useful help.

\section{References}

1. S. Kawka and B.A. van Tiggelen, EPL 89, 11002 (2010). Two errors occur in this work: The atomic Hamiltonian (4) contains an erroneous minus sign in front of $\Delta m$, and Eq. (6) for the interaction $W$ is evaluated without the factor $1 / 2$ in front of the gauge term $\mathbf{B}_{0} \times \mathbf{r}$. Upon restauring these errors, the Casimir momentum proportional to $\alpha$ vanishes, and the $\alpha^{2}$ term becomes leading.

2. J.D. Jackson, Classical Electrodynamics (Wiley, 1975).

3. D.F. Nelson, Phys. Rev. A 44, 3985 (1991).

4. I. Brevik, Phys. Rep. 52, 133 (1979).

5. B.A. van Tiggelen, G.L.J.A. Rikken, Phys. Rev. Lett. 93, 268903 (2004).
6. G.B. Walker and G. Walker, Nature(London) 263, 401 (1976); Can J. Phys. 55, 2121 (1977).

7. G.L.J.A. Rikken and B.A. van Tiggelen, Phys. Rev. Lett. 107, $170401(2011)$.

8. A. Feigel, Phys. Rev. Lett. 92, 020404 (2004).

9. J. Schwinger, Proc. Natl. Acad. Sci USA 90, 958 (1993) and subsequent papers.

10. O.J. Birkeland and I. Brevik, Phys. Rev. E 76, 066605 (2007).

11. I. Brevik, V.N. Marachevsky, and K.A. Milton, Phys. Rev. Lett. 82, 3948 (1999).

12. B.A. van Tiggelen, Eur. Phys. J. D 47, 261 (2008).

13. H. A. Bethe, Phys. Rev. 72, 339 (1947).

14. P.W. Milonni, The Quantum vacuum (Academic Press, San Diego, 1994).

15. M.T. Jaeckel, S. Reynaud, J. Phys. I 3, 1093 (1993).

16. H.B.G. Casimir, D. Polder, Phys. Rev. 73, 360 (1948).

17. O. Dippel, P. Schmelcher, L.S. Cederbaum, Phys. Rev. A. 49, 4415 (1994).

18. C. Cohen-Tannoudji, J. Dupont-Roc, G. Grynberg, Photons et Atomes (Editions du CNRS, Paris, 1987).

19. In this work we will use the SI notation for the static polarizability $\alpha(0)$ used by R.P. Feymann, R.B. Leighton, M. Sands, The Feynman Lectures on Physics (Electromagnetism and Matter) (Addison Wesley, 1981). In that case is the induced atomic polarization $\mathbf{p}_{q}=\varepsilon_{0} \alpha \mathbf{E}$, with $\alpha$ the dimension of a volume, and the induced local polarization density $\mathbf{P}_{q}=\varepsilon_{0} N \alpha \mathbf{E}_{\text {loc }}$ with $N$ the number density of atoms. This convention differs a factor $4 \pi$ from Ref. 21].

20. A. Lagendijk and B.A. van Tiggelen, Phys. Rep. 270(3), 143 (1996).

21. M.M. Bartlett and A.E. Power, J. Phys. A. 2, 419 (1969). 
22. L.D. Landau and E.M. Lifschitz, The Classical Theory of Fields (Pergamon, 1975), section 65.

23. A. Matsumoto, Physica Scripta 44, 154 (1991).

24. R. Loudon, The Quantum Theory of Light second edition (Oxford University Press, 1984).

25. The same assumption of plane waves in the continuous spectrum can be adopted for the static polarizability $\alpha(0)=$ $4 \pi \nu a_{0}^{3}$, for which it is known that $\nu=9 / 2$. The discrete spectrum contributes $\nu_{D}=3.66$. A lower limit $q=0$ would yield $\nu_{C}=2.32$ clearly much too large. The lower limit $q=1 / a_{0}$ yields $\nu_{C}=0.48$, too small. A reasonable choice is somewhat lower than $q=1 / a_{0}$.

\section{A Appendix A}

To leading order, the normalization factor in Eq. (6) will affect the expectation values of $\mathbf{P}$ or $\mathbf{B}_{0} \times \mathbf{r}$. It is therefore sufficient to evaluate it for $\mathbf{Q}_{0}=0, \mathbf{E}_{0}=0$ and $\mathbf{B}_{0}=0$. If we also assume that $m_{1} \gg m_{2}=m_{e}$ we can write

$$
\begin{aligned}
N= & \frac{1}{2}\left\langle 00,0\left|W \frac{1^{\prime}}{\left(\tilde{H}_{0}(\mathbf{p}, \mathbf{Q}, \mathbf{r})+H_{\mathrm{F}}-E_{0}\right)^{2}} W\right| 00,0\right\rangle \\
= & \frac{1}{2} e^{2} \sum_{\mathbf{k} \epsilon} \mathcal{A}_{k}^{2}\langle 0| \epsilon \cdot \mathbf{p} \frac{1}{m_{e}} e^{-i \mathbf{k} \cdot \mathbf{r}} \\
& \frac{1}{\left(\tilde{H}_{0}(\mathbf{p}, \mathbf{Q}=\mathbf{k}, \mathbf{r})+\hbar \omega_{k}-E_{0}\right)^{2}} \epsilon \cdot \mathbf{p} \frac{1}{m_{e}} e^{i \mathbf{k} \cdot \mathbf{r}}|0\rangle \\
= & \frac{e^{2}}{2 m_{e}^{2}} \sum_{\mathbf{k} \epsilon} \mathcal{A}_{k}^{2}\langle 0| \epsilon \cdot \mathbf{p} \\
& \frac{1}{\left(\tilde{H}_{0}(\mathbf{p}+\hbar \mathbf{k}, \mathbf{Q}=\mathbf{k}, \mathbf{r})+\hbar \omega_{k}-E_{0}\right)^{2}} \epsilon \cdot \mathbf{p}|0\rangle
\end{aligned}
$$

If we neglect the $\mathbf{p} \cdot \hbar \mathbf{k} / m_{e}$ term in the denominator, and use $\mathbf{p}|0\rangle=i\left(\hbar / a_{0}\right) \hat{\mathbf{r}}|0\rangle$, one obtains

$$
\begin{aligned}
N= & \frac{e^{2}}{2 m_{e}^{2}} \frac{2}{3} \frac{\hbar^{2}}{a_{0}^{2}} \frac{\hbar}{2 \varepsilon_{0} c_{0}} \\
& \int \frac{d^{3} \mathbf{k}}{(2 \pi)^{3}} \frac{1}{k} \sum_{j}^{\prime} \frac{|\langle 0|\hat{\mathbf{r}}| j\rangle|^{2}}{\left(E_{j}-E_{0}+\hbar^{2} k^{2} / 2 m_{e}+\hbar \omega_{k}\right)^{2}}
\end{aligned}
$$

The $k$-integral has an effective lower limit at $k=\left(E_{j}-\right.$ $\left.E_{0}\right) / \hbar c_{0}$ below which $k$-dependence disappears and we estimate it as,

$$
\begin{aligned}
& \int_{0}^{\frac{E_{j}-E_{0}}{\hbar c_{0}}} d k \frac{k}{\left(E_{j}-E_{0}\right)^{2}}+\int_{\frac{E_{j}-E_{0}}{\hbar c_{0}}}^{\infty} d k \frac{k}{\left(\hbar^{2} k^{2} / 2 m_{e}+\hbar k c_{0}\right)^{2}} \\
\approx & \frac{1}{\left(\hbar c_{0}\right)^{2}}\left(-\log \frac{E_{j}-E_{0}}{\hbar c_{0}}-\frac{1}{2}\right)
\end{aligned}
$$

so that,

$$
N \approx \alpha^{3} \frac{1}{\pi} \sum_{n=2}^{\infty}\left(-\log \frac{E_{n}-E_{0}}{\hbar c_{0}}-\frac{1}{2}\right)|\langle 0|\hat{\mathbf{r}}| n, \ell=1\rangle|^{2}
$$

Using a similar method to evaluate Eq.(23), the sum over Rydberg states at constant logarithm can be evaluated to be 0.336 . The typical value for the logarithm used in calculations of the Lamb shift is -8.35 14. We conclude that $N \approx 0.84 \alpha^{3}$. The continuous spectrum will have a small additional contribution.

\section{B Appendix B}

Let $\mathbf{w} \equiv \mathbf{p}+e(\Delta m / M) \mathbf{B}_{0} \times \mathbf{r} / 2$. We prove that $\left\langle 0, \mathbf{E}_{0}\left|\mathbf{w}^{3}\right| 0, \mathbf{E}_{0}\right\rangle=$ 0 , with notation $\mathbf{w}^{3}:=(\mathbf{w} \cdot \mathbf{w}) \mathbf{w}$. To this end we notice that $\left[\left(\mathbf{w}^{2}\right)^{2}, \mathbf{r}\right]=i \hbar \partial_{\mathbf{p}}\left(\mathbf{w}^{2}\right)^{2}=4 i \hbar \mathbf{w}^{3}$ and that $\tilde{H}_{0}=$ $\mathbf{w}^{2} / 2 \mu+F\left(\mathbf{Q}_{0}, \mathbf{r}\right)$ in the subspace of the eigenvalue $\mathbf{Q}_{0}$ of

P. We see that

$$
\begin{aligned}
\left(\frac{\mathbf{w}^{2}}{2 \mu}\right)^{2} & =\left(\tilde{H}_{0}\right)^{2}-F\left(\mathbf{Q}_{0}, \mathbf{r}\right)^{2}-\frac{\mathbf{w}^{2}}{2 \mu} F\left(\mathbf{Q}_{0}, \mathbf{r}\right)-F\left(\mathbf{Q}_{0}, \mathbf{r}\right) \frac{\mathbf{w}^{2}}{2 \mu} \\
& =\left(\tilde{H}_{0}\right)^{2}-\tilde{H}_{0} F\left(\mathbf{Q}_{0}, \mathbf{r}\right)-F\left(\mathbf{Q}_{0}, \mathbf{r}\right) \tilde{H}_{0}+F\left(\mathbf{Q}_{0}, \mathbf{r}\right)^{2}
\end{aligned}
$$

Since $\left[F\left(\mathbf{Q}_{0}, \mathbf{r}\right), \mathbf{r}\right]=0$ and $\tilde{H}_{0}\left|0, \mathbf{E}_{0}\right\rangle=E_{0}\left|0, \mathbf{E}_{0}\right\rangle$ it follows that

$$
\left\langle 0, \mathbf{E}_{0}\left|\mathbf{w}^{3}\right| 0, \mathbf{E}_{0}\right\rangle=\frac{1}{4 i \hbar}\left\langle 0, \mathbf{E}_{0}\left|\left[\left(\mathbf{w}^{2}\right)^{2}, \mathbf{r}\right]\right| 0, \mathbf{E}_{0}\right\rangle=0
$$

